\title{
Research Article \\ Notes on the Holographic Lifshitz Theory
}

\author{
Chanyong Park \\ Institute for the Early Universe, Ewha Womans University, DaeHyun 11-1, Seoul 120-750, Republic of Korea \\ Correspondence should be addressed to Chanyong Park; cyong21@gmail.com
}

Received 28 November 2013; Accepted 1 February 2014; Published 12 March 2014

Academic Editor: Kingman Cheung

Copyright (C) 2014 Chanyong Park. This is an open access article distributed under the Creative Commons Attribution License, which permits unrestricted use, distribution, and reproduction in any medium, provided the original work is properly cited. The publication of this article was funded by SCOAP ${ }^{3}$.

On the Lifshitz black brane geometry of an Einstein-Maxwell-dilaton gravity, we holographically investigate electric DC conductivities and the role of impurity in a nonrelativistic Lifshitz medium with two different charge carriers, impurity and Lifshitz matter. The conductivity carried by Lifshitz matter is proportional to the square of temperature, while that carried by impurity crucially depends on the bulk coupling parameter $\gamma$. For $\gamma<-2$, impurity at high temperature can change the electric property of the Lifshitz medium significantly so that the Lifshitz matter with impurity can show a totally different electric property from the pure Lifshitz matter.

\section{Introduction}

The AdS/CFT correspondence is a very useful and fascinating tool for understanding the strongly interacting system [1-4]. In the last decade, it has been widely used in studying some universal properties of Quantum Chromodynamics (QCD) and condensed matter systems in the strong coupling regime [5-15]. The asymptotic AdS geometry plays an important role in such investigations because its dual theory is described by the conformal symmetry. Can we generalize the AdS/CFT correspondence to the non-AdS geometry? It is an interesting and also important question in understanding the nonconformal or nonrelativistic condensed matter systems through the holographic methods [16-30]. In this paper, we will study the electric conductivities in the nonrelativistic Lifshitz theory with two kinds of charge carriers.

Following the gauge/gravity duality it was shown that the Einstein-dilaton theory with a Liouville potential corresponds to a relativistic nonconformal theory [31, 32]. In addition, it was also found that the DC conductivity of the dual system can show different behaviors depending on what kind of vector fluctuation is turned on. If a vector fluctuation is not coupled to dilaton, the corresponding DC conductivity in a $2+1$-dimensional relativistic nonconformal theory is temperature independent, while it can have a nontrivial temperature dependence for the vector fluctuation coupled to dilaton. These facts were also checked by using the membrane paradigm [33]. In the similar setup without a Liouville potential, the exact gravity solution has been known as the Lifshitz geometry $[17,18]$. Although the Lifshitz geometry has different scaling in the temporal and spatial coordinates, the generalized scaling symmetry, the so-called hyperscaling symmetry, is still preserved. Due to such a nontrivial scaling, it has been believed that the Lifshitz geometry is dual to a Lifshitz field theory. In particular, when the dynamical exponent is $z=2$, the corresponding dual theory becomes nonrelativistic. In the holographic QCD models for a strongly interacting quark-gluon plasma [34-43], the asymptotic AdS space has been used as the dual geometry. These works were further generalized to the charged AdS geometry, for example, the thermal charged AdS and Reissner-Nordström black brane geometry [36-39]. In these cases, a bulk vector field was identified with matter of the dual theory. Similarly, the bulk vector field of the Lifshitz geometry might be regarded as matter of the Lifshitz field theory. Here, we will simply call it Lifshitz matter or Lifshitz medium. The string theory construction of the nonrelativistic Lifshitz medium was studied in [44] and the binding energy and drag force of external quarks were investigated in [45].

There were many studies on the DC conductivity and superconductivity of the nonrelativistic Lifshitz medium without a dilaton coupling [46-54]. We further investigate 
the DC conductivities of the Lifshitz medium with a nontrivial dilaton coupling, which provides more information for the charge carrier. In this paper, a new vector fluctuation is turned on in the Lifshitz black brane geometry to describe impurity in the nonrelativistic Lifshitz medium. This new vector fluctuation can have a different dilaton coupling from the background gauge field. If we parameterize the different dilaton coupling with $\gamma$, there exists a discrepancy between the results of the membrane paradigm and the Kubo formula for $\gamma \geq 1$. This is due to the change of the asymptotic boundary condition. While the membrane paradigm does not care about the change of the asymptotic boundary condition [55, 56], the Kubo formula crucially depends on it [57-59]. On the other hand, the fluctuation of the background gauge field corresponds to the Lifshitz matter. The DC conductivity carried by it shows totally different behavior from that of impurity because it is coupled to the metric fluctuation through the background gauge field even at quadratic order. The Kubo formula says that the DC conductivity carried by the Lifshitz matter is proportional to the square of temperature. In certain condensed matter systems like semiconductor, impurity is important to explain their electric property. Therefore it is interesting to understand the role of impurity in the medium. We find that at high temperature impurity with $\gamma<-2$ can change the electric property of the nonrelativistic Lifshitz medium significantly.

The rest of the paper is organized as follows. In Section 2, we represent the Lifshitz black brane solution including the manifest hyperscaling symmetry and its thermodynamics with explaining our conventions. In Section 3, the DC conductivities carried by two different charge carriers in the nonrelativistic Lifshitz medium are studied. The results show that impurity with $\gamma<-2$ significantly changes the electric property of the Lifshitz medium at high temperature. Finally, we finish this work with some concluding remarks.

\section{Thermodynamic Properties}

There exist many scale-invariant field theories without the Lorentz invariance near the critical points $[13,15]$. One of such examples is the Lifshitz theory

$$
S[\chi]=\int d^{3} x\left[\left(\partial_{t} \chi\right)^{2}-K\left(\nabla^{2} \chi\right)^{2}\right]
$$

which describes a fixed line parameterized by $K$ with a dynamical exponent $z=2$ [19]. Following the gauge/gravity duality, such a nonrelativistic theory can be mapped to a Lifshitz geometry as a dual gravity. There are several bottomup models, gravity with higher form fields [17] and gravity with a massive gauge field and nondynamical scalar field. These models have been widely investigated by many authors $[19,47,60]$. Another example appears as a geometric solution of the Einstein-Maxwell-dilaton theory. In this paper we will concentrate on the latter case.
Our starting action is the Einstein-Maxwell-dilaton theory with a negative cosmological constant $\Lambda$

$$
\begin{aligned}
S_{\mathrm{EMd}}=\frac{1}{16 \pi G} \int & d^{D} x \sqrt{-g} \\
& \times\left(R-2 \Lambda-\frac{1}{2} \partial_{\mu} \phi \partial^{\mu} \phi-\frac{1}{4} e^{\lambda \phi} F_{\mu \nu} F^{\mu \nu}\right),
\end{aligned}
$$

with

$$
F_{\mu \nu}=\partial_{\mu} A_{\nu}-\partial_{\nu} A_{\mu},
$$

where $\lambda$ is a constant describing the coupling between the gauge field and dilaton. From this action, the black brane geometry satisfying all equations of motion is given by [18, $48,49]$

$$
\begin{gathered}
d s^{2}=-r^{2 z} f(r) d t^{2}+\frac{d r^{2}}{r^{2} f(r)}+r^{2}\left(d x^{2}+d y^{2}\right), \\
\phi(r)=-\frac{4}{\lambda} \log r \\
F_{r t}=\partial_{r} A_{t}=q r^{z+1},
\end{gathered}
$$

with

$$
\begin{gathered}
f(r)=1-\frac{r_{h}^{z+2}}{r^{z+2}}, \\
\lambda=\frac{2}{\sqrt{z-1}}, \\
q=\sqrt{2(z-1)(z+2),} \\
\Lambda=-\frac{(z+1)(z+2)}{2},
\end{gathered}
$$

where $r_{h}$ implies the black brane horizon. It is worthwhile noting that the Lifshitz black brane is not a charged but uncharged solution because the charge $q$ is not a free parameter describing a hair of the black brane. In other words, once the intrinsic parameters of the theory, $\Lambda$ and $\lambda$, are given, the dynamical exponent $z$ and the charge $q$ are automatically determined. In that sense, the Lifshitz black brane geometry might be corresponding to the microcanonical ensemble while the charged black branes are described by a grandcanonical or canonical ensemble. For $z=1$, dilaton and bulk gauge field automatically vanish and the Lifshitz geometry simply reduces to an ordinary AdS geometry where the conformal symmetry is restored. In [61-63], this uncharged Lifshitz black brane was further generalized to the charged case by adding additional gauge fields and their electric and thermodynamic properties were studied.

Note that the above Einstein-Maxwell-dilaton theory preserves a scaling symmetry. When $r$ scales as $\Omega r$, other variables should scale like

$$
\begin{gathered}
r_{h} \longrightarrow \Omega r_{h}, \quad t \longrightarrow \Omega^{-z} t, \quad x \longrightarrow \Omega^{-1} x, \quad y \longrightarrow \Omega^{-1} y, \\
e^{\phi} \longrightarrow \Omega^{-4 / \lambda} e^{\phi}, \quad q \longrightarrow \Omega^{0} q, \\
F_{r t} \longrightarrow \Omega^{z+1} F_{r t}, \quad A_{t} \longrightarrow \Omega^{z+2} A_{t} .
\end{gathered}
$$


These scaling behaviors are different from those of the different Lifshitz models. Then, the time component gauge field satisfying the above scaling is given by

$$
A_{t}=\frac{q}{z+2}\left(r^{z+2}-r_{h}^{z+2}\right)
$$

where the last term corresponds to a new integration constant and we choose a specific value such that the norm of $A_{t}$ is regular even at the black brane horizon. The energy and temperature of this system should scale as the inverse of time, $E \rightarrow \Omega^{z} E$ and $T \rightarrow \Omega^{z} T$. After expanding the metric near the horizon and requiring that there is no conical singularity, the Hawking temperature is determined to be

$$
T=\frac{z+2}{4 \pi} r_{h}^{z}
$$

which shows the correct scaling behavior mentioned previously. The Bekenstein-Hawking entropy is

$$
S=\frac{V_{2}}{4 G} r_{h}^{2}
$$

where $V_{2}$ implies a spatial volume of the boundary space and scales like $V_{2} \rightarrow \Omega^{-2} V_{2}$. Therefore, the Bekenstein-Hawking entropy is invariant under the scaling transformation.

Using the first law of thermodynamics together with the above Hawking temperature and Bekenstein-Hawking entropy, the internal energy $E$ and the free energy $F$ are given by

$$
\begin{gathered}
E=\frac{V_{2}}{8 \pi G} r_{h}^{z+2}, \\
F=-\frac{z V_{2}}{16 \pi G} r_{h}^{z+2} .
\end{gathered}
$$

Using the definition of pressure $P=-\partial F / \partial V_{2}$, we can easily evaluate the equation of state parameter of the Lifshitz black brane

$$
w=\frac{P V_{2}}{E}=\frac{z}{2} .
$$

This implies, according to the gauge/gravity duality, that the dual theory is not conformal except the AdS case with the dynamical exponent $z=1$. Note that it was shown, in the gravity theory with a massive gauge field and nondynamical scalar field (In [19], only the $z=2$ case has been considered and the black brane factor is given by $f(r)=1-r_{h}^{2} / r^{2}$ in our notations, which is different from the present one, $f(r)=1-$ $r_{h}^{4} / r^{4}$ in (5). Although the asymptotic geometries of these two different theories are exactly same, the Hawking temperature due to the difference of inside geometry can have different values. For example, $T=r_{h}^{2} / 2 \pi$ in [19] and $T=r_{h}^{2} / \pi$ in our case.), that the equation of state parameter is given by 1 for $z=2$. The specific heat of this system becomes in terms of temperature

$$
C_{v}=\frac{V_{2}}{2 z G}\left(\frac{4 \pi}{z+2}\right)^{2 / z} T^{2 / z} .
$$

Since it is positive for $z>0$, the dual Lifshitz theory is always thermodynamically stable. In the zero temperature limit $r_{h} \rightarrow 0$, the internal and free energies in (11) become zero. Comparing them with the results at finite temperature, since the free energy at finite temperature is always negative for $z>0$, the Lifshitz black brane is always preferable. This fact implies that there is no Hawking-Page transition. A similar situation also occurs in the relativistic nonconformal theory [32].

\section{DC Conductivities in the Nonrelativistic Lifshitz Medium}

In order to understand macroscopic properties of a nonrelativistic Lifshitz medium, it is useful to investigate the holographic linear response of various fluctuations. Here, we will concentrate on the electric properties of a nonrelativistic Lifshitz theory with two different charge carriers. To do so, we consider a more general Einstein-Maxwell-dilaton theory

$$
\begin{aligned}
& S_{g} \\
& =\frac{1}{16 \pi G} \\
& \quad \times \int d^{D} x \sqrt{-g} \\
& \quad \times\left(R-2 \Lambda-\frac{1}{2} \partial_{\mu} \phi \partial^{\mu} \phi-\frac{1}{4} e^{\lambda \phi} F_{\mu \nu} F^{\mu \nu}-\frac{1}{4} e^{\gamma \phi} H_{\mu \nu} H^{\mu \nu}\right),
\end{aligned}
$$

with

$$
\begin{aligned}
& F_{\mu \nu}=\partial_{\mu} A_{\nu}-\partial_{\nu} A_{\mu}, \\
& H_{\mu \nu}=\partial_{\mu} B_{\nu}-\partial_{\nu} B_{\mu},
\end{aligned}
$$

where $A_{\mu}$ and $B_{\mu}$ are two different $U(1)$ vector fields with different dilaton couplings. In this model, the previous Lifshitz black brane geometry appears as a specific solution for $B_{\mu}=0$. In the dual Lifshitz theory point of view, these two different vector fields correspond to two different matters.

Now, let us turn on vector and metric fluctuations on the Lifshitz black brane geometry. If we denote $a_{\mu}$ and $b_{\mu}$ as fluctuations of $A_{\mu}$ and $B_{\mu}$ respectively, they are governed by the following action at quadratic order:

$$
S_{\text {fluc }}=S_{a}+S_{b}
$$

with

$$
\begin{gathered}
S_{a}=\frac{1}{16 \pi G} \int d^{4} x \sqrt{-g}\left(\mathscr{R}-\frac{e^{\lambda \phi}}{4} f_{\mu \nu} f^{\mu \nu}\right), \\
S_{b}=-\frac{1}{16 \pi G} \int d^{4} x \sqrt{-g} \frac{e^{\gamma \phi}}{4} h_{\mu \nu} h^{\mu \nu},
\end{gathered}
$$

where $f_{\mu \nu}=\partial_{\mu} a_{\nu}-\partial_{\nu} a_{\mu}$ and $h_{\mu \nu}=\partial_{\mu} b_{\nu}-\partial_{\nu} b_{\mu}$. There is no mixing term between $a_{\mu}$ and $b_{\mu}$ at quadratic order, so one can describe those two fluctuations independently. Note that 
since the fluctuation $a_{\mu}$, which is called Lifshitz matter, is coupled to the metric fluctuations through the background gauge field even at quadratic order, one should take into account the metric fluctuations simultaneously. As will be shown, the coupling to the metric fluctuations dramatically changes the DC conductivity carried by the Lifshitz matter. The other fluctuation, $b_{\mu}$, is a new one which corresponds to impurity in the dual Lifshitz field theory. Since impurity has nothing to do with the background gauge field at quadratic order, there is no mixing with the metric fluctuation $[49,64]$. In addition, the coupling parameter $\gamma$ can have an arbitrary number, which may depend on the kind of impurity. From now on we concentrate on the $z=2$ case, which provides an interesting example for a nonrelativistic Lifshitz theory, and take the zero momentum limit because the DC conductivity is well defined even in this limit. In the following sections, we will investigate the DC conductivities carried by impurity and Lifshitz matter with the Kubo formula and show that the different charge carriers lead to the different electric properties.

3.1. DC Conductivity Carried by Impurity. First, let us study the DC conductivity carried by impurity with the Kubo formula. From the action (18) for impurity, the transverse mode, $b_{i}(i=x$ or $y)$, is governed by

$$
0=\partial_{\mu}\left[\sqrt{-g} e^{\gamma \phi} g^{\mu \rho} g^{i \sigma}\left(\partial_{\rho} b_{\sigma}-\partial_{\sigma} b_{\rho}\right)\right]
$$

For $z=2$ and in the zero momentum limit, under the following Fourier mode expansion:

$$
b_{i}(t, r)=\int \frac{d \omega}{2 \pi} e^{-i \omega t} b_{i}(\omega, r) \text {, }
$$

the governing equation simply reduces to

$$
0=b_{i}^{\prime \prime}+\left(\frac{3-2 \gamma}{r}+\frac{f^{\prime}}{f}\right) b_{i}^{\prime}+\frac{\omega^{2}}{r^{6} f^{2}} b_{i} .
$$

At the horizon, $b_{i}$ has two independent solutions

$$
b_{i}(r)=c_{1} f^{ \pm v}
$$

with $v=i\left(\omega / 4 r_{h}^{2}\right)$, where $c_{1}$ is an appropriate normalization constant and the minus or plus sign satisfies the incoming or outgoing boundary condition at the horizon. After choosing an incoming solution, the solution of (21) in the hydrodynamic limit $(\omega \ll T)$ can be perturbatively expanded to

$$
b_{i}(r)=f^{-v}\left[G_{0}(r)+\omega G_{1}(r)\right]+\mathcal{O}\left(\omega^{2}\right) .
$$

In this hydrodynamic expansion, $G_{0}(r), G_{1}(r)$, and all higher order terms should be regular at the horizon which is called a regularity condition. In addition, the above perturbative solution should be reduced to (22) at the horizon, so $G_{0}(r)$ should be a normalization constant $c_{1}$ at the horizon and at the same time the other terms, $G_{1}\left(r_{h}\right)$ and higher order terms, should vanish. We call such a constraint a vanishing condition. Using these two conditions, the perturbative solutions can be exactly determined up to one integration constant

$$
\begin{aligned}
& G_{0}(r)=c_{1}, \\
& G_{1}(r)=c_{3}-\frac{i c_{1}\left[4 \log r-\log \left(r^{4}-r_{h}^{4}\right)\right]}{4 r_{h}^{2}} \\
& \quad-\left(\left(c _ { 4 } \left[{ }_{2} F_{1}\left(1+\gamma, 1,2+\gamma,-\frac{r^{2}}{r_{h}^{2}}\right)\right.\right.\right. \\
& \left.\left.\quad+{ }_{2} F_{1}\left(1+\gamma, 1,2+\gamma, \frac{r^{2}}{r_{h}^{2}}\right)\right] r^{2+2 \gamma}\right) \\
& \left.\quad \times\left(4(1+\gamma) r_{h}^{4}\right)^{-1}\right),
\end{aligned}
$$

with

$$
\begin{aligned}
c_{3}=\left(i c_{1}[\right. & -\mathrm{PG}\left(0,1+\frac{\gamma}{2}\right)+\mathrm{PG}\left(0, \frac{1+\gamma}{2}\right) \\
& +2\{\mathrm{EG}-\log 2+\mathrm{PG}(0,1+\gamma)\}]) \times\left(8 r_{h}^{2}\right)^{-1},
\end{aligned}
$$$$
c_{4}=-i c_{1} r_{h}^{-2 \gamma}
$$

where PG and EG mean the poly gamma and Euler gamma function, respectively. In order to determine the remaining integration constant $c_{1}$, we should impose another boundary condition. At the asymptotic boundary, the vector fluctuation $b_{i}$ has the following asymptotic expansion:

$$
b_{i}(r)=b_{1}+b_{2} r^{2 \gamma-2}
$$

where $b_{1}$ (or $b_{2}$ ) is a constant determined by the asymptotic boundary condition.

3.1.1. For $\gamma<1$. If $\gamma$ is smaller than 1 , the asymptotic behavior of $b_{i}(r)$ is determined by the first term $b_{1}$. According to the usual gauge/gravity duality, the first coefficient corresponds to the source while the second describes the vacuum expectation value (vev) of the dual operator. In this case, it is natural to impose the Dirichlet boundary condition like

$$
b_{0} \equiv \lim _{r_{0} \rightarrow \infty} b_{i}\left(r_{0}\right)
$$

where $r_{0}$ implies an appropriate UV cutoff of the dual theory and $b_{0}$ corresponds to the boundary value of $b_{i}$ which is equal to $b_{1}$ for $\gamma<1$. Comparing the asymptotic expansion of the perturbative solution (23) with the above boundary condition (27), the remaining integration constant $c_{1}$ can be rewritten in terms of the boundary value $b_{0}$ as

$$
\begin{aligned}
& c_{1}=8 i r_{h}^{2} b_{0} \\
& \times\left(8 i r_{h}^{2}+\omega\left[\mathrm{HN}\left(\frac{\gamma}{2}\right)-\mathrm{HN}\left(\frac{-1+\gamma}{2}\right)\right.\right. \\
& \left.\left.\quad-2 \mathrm{HN}(\gamma)+2 \log 2+2 \pi \tan \left(\frac{\pi \gamma}{2}\right)\right]\right)^{-1},
\end{aligned}
$$

where HN means a harmonic number. 
The boundary action corresponding to the on-shell action of (18) is given by

$$
\begin{aligned}
S_{B} & =-\frac{1}{16 \pi G} \int_{r=r_{0}} d^{3} x \sqrt{-g} e^{\gamma \phi} g^{r r} g^{i i} b_{i} b_{i}^{\prime} \\
& \approx-\frac{1}{16 \pi G} \int d^{3} x r_{h}^{-2 \gamma} r_{0}^{3-2 \gamma} b_{0} b_{i}^{\prime} .
\end{aligned}
$$

This result shows that the finite contributions to the boundary action can come from $b_{i}^{\prime} \sim r_{0}^{-3+2 \gamma}$ when $r_{0} \rightarrow \infty$. Since the asymptotic expansion of $b_{i}^{\prime}$ from (23) has

$$
b_{i}^{\prime}=-\frac{i c_{1} \omega}{r_{h}^{2 \gamma}} \frac{1}{r_{0}^{3-2 \gamma}}+\mathcal{O}\left(\frac{1}{r^{5}}\right)
$$

the current-current retarded Green function $[58,59]$ results in

$$
\left\langle J^{i} J^{i}\right\rangle=\frac{i \omega}{16 \pi G} \frac{1}{r_{h}^{2 \gamma}}+\mathcal{O}\left(\omega^{2}\right),
$$

where (28) is used. Finally, the DC conductivity from the Kubo formula reads

$$
\sigma_{\mathrm{DC}} \equiv \lim _{\omega \rightarrow 0} \frac{\left\langle J^{i} J^{i}\right\rangle}{i \omega}=\frac{1}{16 \pi^{\gamma+1} G} \frac{1}{T^{\gamma}} .
$$

3.1.2. For $\gamma \geq 1$. Let us take into account the case with $\gamma \geq 1$. In this case, the DC conductivity carried by impurity shows a totally different behavior compared with the previous case because the interpretation of the asymptotic solution should be modified. From now on, we will concentrate on the case with $\gamma=2$ for later comparison with the DC conductivity carried by Lifshitz matter.

Similar to (23), the perturbative expansion of solution in the zero momentum limit is given by

$$
b_{i}(r)=f^{-\nu}\left[G_{0}(r)+\omega G_{1}(r)+\omega^{2} G_{2}(r)\right]+\mathcal{O}\left(\omega^{3}\right),
$$

where $v=i\left(\omega / 4 r_{h}^{2}\right)$. In this case, $\omega^{2} G_{2}(r)$ is important to determine the DC conductivity unlike the previous case. The solutions, $G_{0}(r)$ and $G_{1}(r)$, satisfying the regularity and vanishing condition at the horizon are

$$
\begin{aligned}
G_{0}(r)= & c_{1}, \\
G_{1}(r)=- & {\left[\frac{i}{2 r_{h}^{4}} r^{2}-\frac{\pi+2 i(1-\log 2)}{4 r_{h}^{2}}\right.} \\
& \left.+\frac{i}{r_{h}^{2}} \log r-\frac{i}{2 r_{h}^{2}} \log \left(r^{2}+r_{h}^{2}\right)\right] c_{1} .
\end{aligned}
$$

After inserting these two solutions into (21), we can find the analytic form of $G_{2}(r)$ which has the following expansion near the horizon:

$$
\begin{aligned}
& G_{2}(r) \\
& =\frac{12 c_{6} r_{h}^{6}-c_{1}(6 \log 2-10-3 \pi i)}{48 r h^{4}} \log \left(r-r_{h}\right) \\
& +c_{5}+\frac{r_{h}^{2}\left(2+\pi i-\log r_{h}\right)}{4} c_{6} \\
& +\frac{5 \pi^{2}+8\left(9-10 \log 2+3(\log 2)^{2}\right)+12 \pi i(3+2 \log 2)}{96 r_{h}^{4}} c_{1} \\
& +\frac{(-20+42 \pi i+60 \log 2) \log r_{h}+48\left(\log r_{h}\right)^{2}}{96 r_{h}^{4}} c_{1}+\mathcal{O}\left(r-r_{h}\right) .
\end{aligned}
$$

Again, imposing the regularity and vanishing condition at the horizon, $c_{5}$ and $c_{6}$ are fixed to be

$$
\begin{aligned}
c_{5}= & -\left[\frac{32+4 \pi i+11 \pi^{2}-56 \log 2+36 \pi i \log 2+24(\log 2)^{2}}{96 r_{h}^{4}}\right. \\
& \left.+\frac{48(\pi i+\log 2) \log r_{h}}{96 r_{h}^{4}}+\frac{\left(\log r_{h}\right)^{2}}{2 r_{h}^{4}}\right] c_{1}, \\
c_{6}= & \frac{6 \log 2-10-3 \pi i}{12 r_{h}^{6}} c_{1} .
\end{aligned}
$$

Before calculating the conductivity, it is worth to note that for $\gamma \geq 1$ the second terms in (26) are more dominant when determining the asymptotic behavior of impurity. This implies that the previous Dirichlet boundary condition in (27) cannot fix $b_{1}$, so we need to modify the asymptotic boundary condition. A natural choice is choosing the second coefficient $b_{2}$ as a source rather than the first and then fixing it by an appropriate boundary condition. Following this strategy, the appropriate asymptotic boundary condition for $\gamma \geq 1$ should be [57]

$$
b_{0}=\lim _{r_{0} \rightarrow \infty} \frac{b_{i}\left(r_{0}\right)}{r_{0}^{2 \gamma-2}} .
$$

Especially, for $\gamma=2$ the boundary condition reduces to

$$
b_{0}=\lim _{r \rightarrow \infty} \frac{b_{i}\left(r_{0}\right)}{r_{0}^{2}} .
$$

Using (34) and (36) together with the exact solution for $G_{2}(r)$, we can easily find the asymptotic expansion of $b_{i}(r)$ up to $\omega^{2}$ and comparing it with the boundary condition in (38) determines $c_{1}$ in terms of $b_{0}$

$$
c_{1}=\frac{24 i r_{h}^{4}}{\omega\left[12 r_{h}^{2}+i \omega(6 \log 2-10-3 \pi i)\right]} b_{0} .
$$


At first glance, it looks extraordinary because $c_{1}$ is proportional to $\omega^{-1}$. However, near the horizon it still becomes a solution whose normalization constant is proportional to $\omega^{-1}$.

Since the boundary action for $\gamma=2$ is given by

$$
S_{B}=-\frac{1}{16 \pi G} \int d^{3} x r_{0} b_{0} b_{i}^{\prime},
$$

only $b_{i}^{\prime} \sim r_{0}^{-1}$ can provide the finite contribution to the boundary action. If $(\partial / \partial r)\left(f^{-v} G_{0}\right)$ or $(\partial / \partial r)\left(f^{-v} \omega G_{1}\right)$ contains such a term, the DC conductivity diverges with $\omega^{-2}$ or $\omega^{-1}$, respectively, because $c_{1} \sim 1 / \omega$. This fact says that the finite contribution to the DC conductivity is determined not by $\omega G_{1}$ but by $\omega^{2} G_{2}$. In the asymptotic region $\left(r_{0} \rightarrow \infty\right)$, the expansion of $b_{i}^{\prime}$ has the following form:

$$
b_{i}^{\prime}=\# r_{0}+\frac{\#}{r_{0}^{3}}+\mathcal{O}\left(\frac{1}{r_{0}^{5}}\right)
$$

where \# implies a certain number. This result shows that $b_{i}^{\prime}$ has no term proportional to $r_{0}^{-1}$ so that the finite part of the resulting boundary action becomes zero. Consequently, the DC conductivity carried by impurity for $\gamma=2$ vanishes:

$$
\sigma_{\mathrm{DC}}=0 \text { for } \gamma=2 \text {. }
$$

Before concluding this section, there is an important remark. The result in (42) is totally different from that of the membrane paradigm. In the membrane paradigm, the DC conductivity especially for impurity can be represented only by the horizon quantities. The resulting form for $z=2$ is $[33,55,56]$

$$
\sigma_{\mathrm{DC}}=\left.\frac{e^{\gamma \phi}}{16 \pi G} \sqrt{\frac{g}{g_{t t} g_{r r}}} g^{i i}\right|_{r_{h}}=\frac{1}{16 \pi^{\gamma+1} G} \frac{1}{T^{\gamma}} .
$$

This is exactly the form obtained in the previous section for $\gamma<1$. However, it is not consistent with the result for $\gamma=2$. The reason is that in the Kubo formula the asymptotic boundary condition is modified for $\gamma \geq 1$ while the membrane paradigm does not care about the asymptotic behavior of the solution. Because of that, the membrane paradigm is consistent with the Kubo formula only for $\gamma<1$. Following the spirit of the AdS/CFT correspondence, physical quantities like the correlation functions of the dual field theory should be holographically governed by bulk field fluctuations near the asymptotic region. In this sense, the Kubo formula results seem to be more fundamental.

3.2. DC Conductivity Carried by Lifshitz Matter. Now, consider Lifshitz matter instead of impurity. Generally, if there exists a background gauge field, the transverse mode of its fluctuation should be coupled to the shear mode of the metric fluctuations [40]. Therefore, in order to study the linear response of such an $U(1)$ gauge field, we should also consider the metric shear mode. The equations governing the transverse and shear modes can be derived from (17). After the Fourier mode expansion

$$
\begin{aligned}
& g_{t}^{i}(t, r)=\int \frac{d \omega}{2 \pi} e^{-i \omega t} g_{t}^{i}(\omega, r), \\
& a_{i}(t, r)=\int \frac{d \omega}{2 \pi} e^{-i \omega t} a_{i}(\omega, r),
\end{aligned}
$$

the governing equations for shear modes reduce to

$$
\begin{gathered}
0=g_{t}^{i^{\prime}}+\frac{q}{r^{5-z}} a_{i}, \\
0=g_{t}^{i^{\prime \prime}}+\frac{(5-z)}{r} g_{t}^{i^{\prime}}+\frac{q}{r^{5-z}} a_{i}^{\prime},
\end{gathered}
$$

where $g_{t}^{i}$ and $a_{i}$ imply $g_{t}^{i}(\omega, r)$ and $a_{i}(\omega, r)$. The first equation (45) is a constraint which automatically satisfies the second equation (46). The equation governing the transverse modes leads to

$$
0=a_{i}^{\prime \prime}+\frac{r f^{\prime}+(z-3) f}{r f} a_{i}^{\prime}+\frac{q r^{3-z}}{f} g_{t}^{i^{\prime}}+\frac{w^{2}}{r^{2+2 z} f^{2}} a_{i} .
$$

Inserting the constraint into (47), the decoupled differential equation of the transverse mode becomes

$$
0=a_{i}^{\prime \prime}+\frac{r f^{\prime}+(z-3) f}{r f} a_{i}^{\prime}+\left(\frac{w^{2}}{r^{2 z+2} f^{2}}-\frac{q^{2}}{r^{2} f}\right) a_{i} .
$$

At the horizon, due to vanishing of $f, a_{i}$ should have the following two independent solutions up to an overall normalization constant

$$
a_{i} \sim f^{ \pm i\left(\omega / 4 r_{h}^{2}\right)}
$$

where the plus or minus sign again implies the outgoing or incoming mode. In the hydrodynamic limit, after taking only the incoming part, the near horizon solution of $a_{i}$ can be expanded into

$$
a_{i}=f^{-i\left(\omega / 4 r_{h}^{2}\right)}\left[G_{0}(r)+\omega G_{1}(r)\right]+\mathcal{O}\left(\omega^{2}\right),
$$

where $G_{0}(r)$ and $G_{1}(r)$ should be regular functions at the black brane horizon. After substituting the perturbative expansion into (48), one can solve it order by order. For $z=2$, $G_{0}$ has the following exact solution at $\omega^{0}$ order:

$$
G_{0}=c_{1}\left(r^{4}+r_{h}^{4}\right)-\frac{c_{2} r^{2}}{8 r_{h}^{4}}+\frac{c_{2}\left(r^{4}+r_{h}^{4}\right)}{8 r_{h}^{6}} \operatorname{arctanh}\left(\frac{r^{2}}{r_{h}^{2}}\right),
$$

where $c_{1}$ and $c_{2}$ are two integration constants. Imposing the regularity condition at the horizon, $c_{2}=0$ because $\operatorname{arctanh}\left(r^{2} / r_{h}^{2}\right)$ diverges. Consequently,

$$
G_{0}=c_{1}\left(r^{4}+r_{h}^{4}\right) \text {. }
$$


Using this result, at next order of $\omega$ the solution of $G_{1}$ is given by

$$
\begin{aligned}
G_{1}= & c_{3}\left(r^{4}+r_{h}^{4}\right)-\frac{c_{4} r^{2}}{8 r_{h}^{4}}-\frac{i c_{1}\left(r^{4}+r_{h}^{4}\right) \log r}{r_{h}^{2}} \\
& +\frac{\left(r^{4}+r_{h}^{4}\right)\left(c_{4}+i 4 c_{1} r_{h}^{4}\right) \log \left(r^{2}+r_{h}^{2}\right)}{16 r_{h}^{6}} \\
& -\frac{\left(r^{4}+r_{h}^{4}\right)\left(c_{4}-i 4 c_{1} r_{h}^{4}\right) \log \left(r^{2}-r_{h}^{2}\right)}{16 r_{h}^{6}} .
\end{aligned}
$$

Since the last term diverges at the horizon, the regularity condition determines

$$
c_{4}=i 4 c_{1} r_{h}^{4}
$$

In addition, the vanishing condition fixes the rest integration constant as

$$
c_{3}=-\frac{i c_{1}(2 \log 2-1)}{4 r_{h}^{2}} .
$$

Now, let us consider the asymptotic behavior of the solution. Unlike the impurity case, the asymptotic behavior of $a_{i}$ is totally different from that of $b_{i}$ due to the nontrivial mixing with the shear mode. From (48), the asymptotic behavior for $z=2$ is governed by

$$
0=a_{i}^{\prime \prime}-\frac{1}{r} a_{i}^{\prime}-\frac{8}{r^{2}} a_{i},
$$

where the last term is originated from the shear mode. At the asymptotic boundary, $a_{i}$ has the following perturbative solution:

$$
a_{i}=a_{1} r^{4}+\frac{a_{2}}{r^{2}}
$$

where $a_{1}$ is a constant to be determined by the asymptotic boundary condition. One can identify the coefficients of the nonnormalizable and normalizable modes, $a_{1}$ and $a_{2}$, with the source and expectation value of the dual operator, respectively. In order to fix the boundary value of $a_{i}$, we impose the following boundary condition at the asymptotic boundary:

$$
a_{0} \equiv \lim _{r \rightarrow \infty} \frac{a_{i}(r)}{r^{4}}
$$

Then, the integration constant $c_{1}$ is fixed in terms of the boundary value $a_{0}$ to be

$$
c_{1}=\frac{4 r_{h}^{2} a_{0}}{4 r_{h}^{2}-i \omega(2 \log 2-1)} .
$$

From the action (17), the boundary term of the Lifshitz matter becomes

$$
\begin{aligned}
S_{B} & =-\frac{1}{16 \pi G} \int d^{3} x \sqrt{-g} e^{\lambda \phi} g^{r r} g^{i i} a_{i} \partial_{r} a_{i} \\
& =-\frac{1}{16 \pi G} \int d^{3} x r_{0}^{3} a_{0} \partial_{r} a_{i},
\end{aligned}
$$

where one can see that the finite part of the retarded Green function comes from $\partial_{r} a_{i} \sim r_{0}^{-3}$. When ignoring the divergent parts corresponding to the contact terms, the DC conductivity carried by the Lifshitz matter leads to

$$
\sigma_{\mathrm{DC}}=\frac{\pi}{12 G} T^{2}
$$

This result shows that the DC conductivity carried by a Lifshitz matter is totally different from that carried by impurity for $\gamma=2$.

Impurity in semiconductor dramatically changes the electric property of matter from an insulator at low temperature to a conductor at high temperature. In many condensed matter systems like a semiconductor, impurity plays a crucial role in physics so that it is important to understand the effect of such impurity. In this paper, we holographically realize impurity by turning on a different bulk vector fluctuation in the Lifshitz black brane geometry. In the dual theory point of view, it corresponds to inserting impurity into the Lifshitz medium. Depending on the kind of impurity parameterized by $\gamma$, it can dramatically change the electric property of the Lifshitz matter. For instance, if impurity is characterized by $\gamma<-2$, the DC conductivity of the Lifshitz matter is not affected by impurity at low temperature. However, at high temperature the DC conductivity of impurity is dominant so that the electric property of the Lifshitz matter with impurity can show totally different behavior from the pure Lifshitz matter.

\section{Discussion}

We have investigated thermodynamics and hydrodynamics of the nonrelativistic Lifshitz medium with two types of charge carriers. To do so, we considered Lifshitz black brane solutions of the Einstein-Maxwell-dilaton gravity. There are several models describing the same asymptotic Lifshitz geometry. Although all models give rise to the same thermodynamic relation and the equation of state parameter, the details of the thermodynamic quantities are different due to the different interior metric. In this paper, we clarified the scaling behaviors of all fields and thermodynamic quantities of the Lifshitz black brane geometry derived from the Einstein-Maxwell-dilaton gravity.

After that, we have studied the holographic responses of two types of vector fluctuations which describe the DC conductivities carried by two different charge carriers, impurity and the Lifshitz matter, in the nonrelativistic Lifshitz medium. In this case, to realize impurity on the dual gravity we have turned on another vector fluctuation in the Lifshitz black brane geometry. For the DC conductivity carried by impurity, there are two different methods, the Kubo formula and membrane paradigm. In the nonrelativistic Lifshitz medium, these two formulas give the same result for $\gamma<$ 1 but we found that there exists a marked discrepancy for $\gamma \geq 1$. For $\gamma \geq 1$, one should change the asymptotic boundary condition to find the DC conductivity. This fact can be consistently imposed in the Kubo formula, while the membrane paradigm, because it is described by only 
information at the horizon, cannot know this fact. In that sense, the Kubo formula looks more fundamental.

For the DC conductivity carried by the Lifshitz matter, its dual vector fluctuation is coupled to the metric fluctuation through the background gauge field. The coupling with the metric fluctuation leads to a nontrivial DC conductivity proportional to the square of temperature for $z=2$. Like an example of semiconductor, we found that impurity with $\gamma<-2$ can crucially modify the electric property of the nonrelativistic Lifshitz matter at high temperature.

Finally, the Einstein-Maxwell-dilaton theory studied in this paper has a negative cosmological constant and allows the Lifshitz geometry with a hyperscaling symmetry. If one modifies this negative cosmological constant to a Liouvilletype of the dilaton potential, the deformed theory gives rise to the hyperscaling violation geometry in which both the scaling and boundary Lorentz symmetries are broken. In this set-up, it is interesting to investigate the various thermodynamic and hydrodynamic properties of the dual nonrelativistic theory without a scaling symmetry. We hope to report some interesting results on these issues in future works.

\section{Conflict of Interests}

The author declares that there is no conflict of interests regarding the publication of this paper.

\section{Acknowledgments}

This work has been supported by the WCU Grant no. R3210130 and the Research Fund no. 1-2008-2935-001-2 by Ewha Womans University. Chanyong Park was also supported by Basic Science Research Program through the National Research Foundation of Korea (NRF) funded by the Ministry of Education (NRF-2013R1A1A2A10057490).

\section{References}

[1] J. M. Maldacena, "The large- $N$ limit of superconformalfield theories and supergravity," Advances in Theoretical Mathematical Physics, vol. 2, pp. 231-252, 1998.

[2] S. S. Gubser, I. R. Klebanov, and A. M. Polyakov, "Gauge theory correlators from non-critical string theory," Physics Letters B, vol. 428, no. 1-2, pp. 105-114, 1998.

[3] E. Witten, "Anti de Sitter space and holography," Advances in Theoretical Mathematical Physics, vol. 2, pp. 253-291, 1998.

[4] E. Witten, "Anti-de Sitter space, thermal phase transition, and confinement in gauge theories," Advances in Theoretical Mathematical Physics, vol. 2, pp. 505-532, 1998.

[5] T. Sakai and S. Sugimoto, "Low energy hadron physics in holographic QCD," Progress of Theoretical Physics, vol. 113, no. 4, pp. 843-882, 2005.

[6] T. Sakai and S. Sugimoto, "More on a holographic dual of QCD," Progress of Theoretical Physics, vol. 114, no. 5, pp. 1083-1118, 2005.

[7] J. Erlich, E. Katz, D. T. Son, and M. A. Stephanov, "QCD and a holographic model of hadrons," Physical Review Letters, vol. 95, no. 26, Article ID 261602, 4 pages, 2005.
[8] A. Karch, E. Katz, D. T. Son, and M. A. Stephanov, "Linear confinement and AdS/QCD," Physical Review D, vol. 74, no. 1, Article ID 015005, 7 pages, 2006.

[9] C. P. Herzog, "Holographic prediction for the deconfinement temperature," Physical Review Letters, vol. 98, no. 9, Article ID 091601, 4 pages, 2007.

[10] S. A. Hartnoll, C. P. Herzog, and G. T. Horowitz, "Building a holographic superconductor," Physical Review Letters, vol. 101, no. 3, Article ID 031601, 4 pages, 2008.

[11] S. A. Hartnoll, C. P. Herzog, and G. T. Horowitz, "Holographic superconductors," Journal of High Energy Physics, vol. 2008, no. 12, article 015, 2008.

[12] G. T. Horowitz and J. Polchinski, "Gauge/gravity duality," in Approaches to Quantum Gravity, D. Oriti, Ed., pp. 169-186, http://arxiv.org/abs/gr-qc/0602037.

[13] S. A. Hartnoll, "Lectures on holographic methods for condensed matter physics," Classical and Quantum Gravity, vol. 26, no. 22, Article ID 224002, 2009.

[14] S. Sachdev, "Condensed matter and AdS/CFT," in From Gravity to Thermal Gauge Theories: The AdS/CFT Correspondence, vol. 828 of Lecture Notes in Physics, pp. 273-311, Springer, Berlin, Germany, 2011.

[15] S. Sachdev and M. Müller, "Quantum criticality and black holes," Journal of Physics, vol. 21, no. 16, Article ID 164216, 2009.

[16] P. Koroteev and M. Libanov, "On existence of self-tuning solutions in static braneworlds without singularities," Journal of High Energy Physics, vol. 2008, no. 2, article 104, 2008.

[17] S. Kachru, X. Liu, and M. Mulligan, "Gravity duals of Lifshitzlike fixed points," Physical Review D, vol. 78, no. 10, Article ID 106005, 8 pages, 2008.

[18] M. Taylor, "Non-relativistic holography," http://arxiv.org/abs/ 0812.0530 .

[19] K. Balasubramanian and J. McGreevy, "An analytic Lifshitz black hole," Physical Review D, vol. 80, no. 10, Article ID 104039, 8 pages, 2009.

[20] S. F. Ross and O. Saremi, "Holographic stress tensor for nonrelativistic theories," Journal of High Energy Physics, vol. 2009, no. 9, article 009, 2009.

[21] K. Goldstein, N. Iizuka, S. Kachru, S. Prakash, S. P. Trivedi, and A. Westphal, "Holography of dyonic dilaton black branes," Journal of High Energy Physics, vol. 2010, no. 10, article 027, 2010.

[22] C. Charmousis, B. Goutéraux, B. S. Kim, E. Kiritsis, and R. Meyer, "Effective holographic theories for low-temperature condensed matter systems," Journal of High Energy Physics, vol. 2010, no. 11, article 151, 2010.

[23] B. Goutéraux and E. Kiritsis, "Generalized holographic quantum criticality at finite density," Journal of High Energy Physics, vol. 2011, no. 12, article 36, 2011.

[24] E. Kiritsis, "Lorentz violation, gravity, dissipation and holography," Journal of High Energy Physics, vol. 2013, no. 1, article 30, 2013.

[25] X. Dong, S. Harrison, S. Kachru, G. Torroba, and H. Wang, "Aspects of holography for theories with hyperscaling violation," Journal of High Energy Physics, vol. 2012, no. 6, article 41, 2012.

[26] L. Huijse, S. Sachdev, and B. Swingle, "Hidden Fermi surfaces in compressible states of gauge-gravity duality," Physical Review $B$, vol. 85, no. 3, Article ID 035121, 14 pages, 2012.

[27] E. Perlmutter, "Hyperscaling violation from supergravity," Journal of High Energy Physics, vol. 2012, no. 6, article 165, 2012. 
[28] K. Narayan, "AdS null deformations with inhomogeneities," Physical Review D, vol. 86, no. 12, Article ID 126004, 12 pages, 2012.

[29] I. Kanitscheider and K. Skenderis, "Universal hydrodynamics of non-conformal branes," Journal of High Energy Physics, vol. 2009, no. 4, article 062, 2009.

[30] B. Gouteraux, J. Smolic, M. Smolic, K. Skenderis, and M. Taylor, "Holography for Einstein-Maxwell-dilaton theories from generalized dimensional reduction," Journal of High Energy Physics, vol. 2012, no. 1, article 89, 2012.

[31] S. Kulkarni, B.-H. Lee, C. Park, and R. Roychowdhury, "Nonconformal hydrodynamics in Einstein-dilaton theory," Journal of High Energy Physics, vol. 2012, no. 9, article 4, 2012.

[32] S. Kulkarni, B.-H. Lee, J.-H. Oh, C. Park, and R. Roychowdhury, "Transport in non-conformal holographic fluids," Journal of High Energy Physics, vol. 2013, no. 3, article 149, 2013.

[33] C. Park, "Holographic aspects of a relativistic non-conformal theory," Advances in High Energy Physics, vol. 2013, Article ID 389541, 16 pages, 2013.

[34] J. M. Maldacena, "Wilson loops in large $N$ field theories," Physical Review Letters, vol. 80, no. 22, pp. 4859-4862, 1998.

[35] S.-J. Rey and J.-T. Yee, "Macroscopic strings as heavy quarks: large- $N$ gauge theory and anti-de Sitter supergravity," The European Physical Journal C, vol. 22, no. 2, pp. 379-394, 2001.

[36] B.-H. Lee, C. Park, and S.-J. Sin, "A dual geometry of the hadron in dense matter," Journal of High Energy Physics, vol. 2009, no. 7, article 087, 2009.

[37] C. Park, "Dissociation of a heavy meson in the quark medium," Physical Review D, vol. 81, no. 4, Article ID 045009, 7 pages, 2010.

[38] C. Park, "Holographic symmetry energy of the nuclear matter," Physics Letters B, vol. 708, no. 3-5, pp. 324-329, 2012.

[39] B.-H. Lee, S. Nam, C. Park, and S. Mamedov, "Holographic meson mass splitting in the nuclear matter," Journal of High Energy Physics, vol. 2013, no. 8, article 45, 2013.

[40] X.-H. Ge, Y. Matsuo, F.-W. Shu, S.-J. Sin, and T. Tsukioka, "Density dependence of transport coefficients from holographic hydrodynamics," Progress Theoretical Physics, vol. 120, no. 5, pp. 833-863, 2008.

[41] S. S. Gubser, "Drag force in AdS/CFT," Physical Review D, vol. 74, no. 12, Article ID 126005, 4 pages, 2006.

[42] Y. Kim, B.-H. Lee, C. Park, and S.-J. Sin, "The effect of gluon condensate on holographic heavy quark potential," Physical Review D, vol. 80, no. 10, Article ID 105016, 7 pages, 2009.

[43] Y. Ko, B.-H. Lee, and C. Park, "Meson spectra in a gluon condensate background," Journal of High Energy Physics, vol. 2010, no. 4, article 37, 2010.

[44] K. Balasubramanian and K. Narayan, "Lifshitz spacetimes from AdS null and cosmological solutions," Journal of High Energy Physics, vol. 2010, no. 8, article 14, 2010.

[45] K. B. Fadafan, "Drag force in asymptotically Lifshitz spacetimes," http://arxiv.org/abs/0912.4873.

[46] R. B. Mann, "Lifshitz topological black holes," Journal of High Energy Physics, vol. 2009, no. 6, article 75, 2009.

[47] G. Bertoldi, B. A. Burrington, and A. Peet, "Black holes in asymptotically Lifshitz spacetimes with arbitrary critical exponent," Physical Review D, vol. 80, no. 12, Article ID 126003, 13 pages, 2009.

[48] D.-W. Pang, "A note on black holes in asymptotically Lifshitz spacetime," http://arxiv.org/abs/0905.2678.
[49] D.-W. Pang, "Conductivity and diffusion constant in Lifshitz backgrounds," Journal of High Energy Physics, vol. 2010, no. 1, article 120, 2010.

[50] E. J. Brynjolfsson, U. H. Danielsson, L. Thorlacius, and T. Zingg, "Holographic superconductors with Lifshitz scaling," Journal of Physics A, vol. 43, no. 6, Article ID 065401, 2010.

[51] E. Ayon-Beato, A. Garbarz, G. Giribet, and M. Hassaine, "Lifshitz black hole in three dimensions," Physical Review D, vol. 80, no. 10, Article ID 104029, 4 pages, 2009.

[52] R.-G. Cai, Y. Liu, and Y.-W. Sun, "A Lifshitz black hole in four dimensional $R^{2}$ gravity," Journal of High Energy Physics, vol. 2009, no. 10, article 80, 2009.

[53] S.-J. Sin, S.-S. Xu, and Y. Zhou, "Holographic superconductor for a Lifshitz fixed point," International Journal of Modern Physics A, vol. 26, no. 26, pp. 4617-4631, 2011.

[54] X.-H. Ge, B. Wang, S.-F. Wu, and G.-H. Yang, "Analytical study on holographic superconductors in external magnetic field," Journal of High Energy Physics, vol. 2010, no. 8, article 108, 2010.

[55] P. Kovtun, D. T. Son, and A. O. Starinets, "Holography and hydrodynamics: diffusion on stretched horizons," Journal of High Energy Physics, vol. 2003, no. 10, article 64, 2003.

[56] N. Iqbal and H. Liu, "Universality of the hydrodynamic limit in AdS/CFT and the membrane paradigm," Physical Review D, vol. 79, no. 2, Article ID 025023, 15 pages, 2009.

[57] D. T. Son and A. O. Starinets, "Minkowski-space correlators in AdS/CFT correspondence: recipe and applications," Journal of High Energy Physics, vol. 2002, no. 9, article 042, 2002.

[58] G. Policastro, D. T. Son, and A. O. Starinets, "From AdS/CFT correspondence to hydrodynamics," Journal of High Energy Physics, vol. 2002, no. 9, article 043, 2002.

[59] G. Policastro, D. T. Son, and A. O. Starinets, "From AdS/CFT correspondence to hydrodynamics. II. Sound waves," Journal of High Energy Physics, vol. 2002, no. 12, article 054, 2002.

[60] Y. Korovin, K. Skenderis, and M. Taylor, "Lifshitz as a deformation of Anti-de Sitter," Journal of High Energy Physics, vol. 2013, no. 8, article 026, 2013.

[61] J. Tarrio and S. Vandoren, "Black holes and black branes in Lifshitz spacetimes," Journal of High Energy Physics, vol. 2011, no. 9, article 017, 2011.

[62] J.-R. Sun, S.-Y. Wu, and H.-Q. Zhang, "Novel features of the transport coefficients in Lifshitz black branes," Physical Review $D$, vol. 87, no. 8, Article ID 086005, 14 pages, 2013.

[63] J.-R. Sun, S.-Y. Wu, and H.-Q. Zhang, "Mimic the optical conductivity in disordered solids via gauge/gravity duality," Physics Letters B, vol. 729, pp. 177-184, 2014.

[64] B.-H. Lee, S. Nam, D.-W. Pang, and C. Park, "Conductivity in an anisotropic background," Physical Review D, vol. 83, no. 6, Article ID 066005, 12 pages, 2011. 

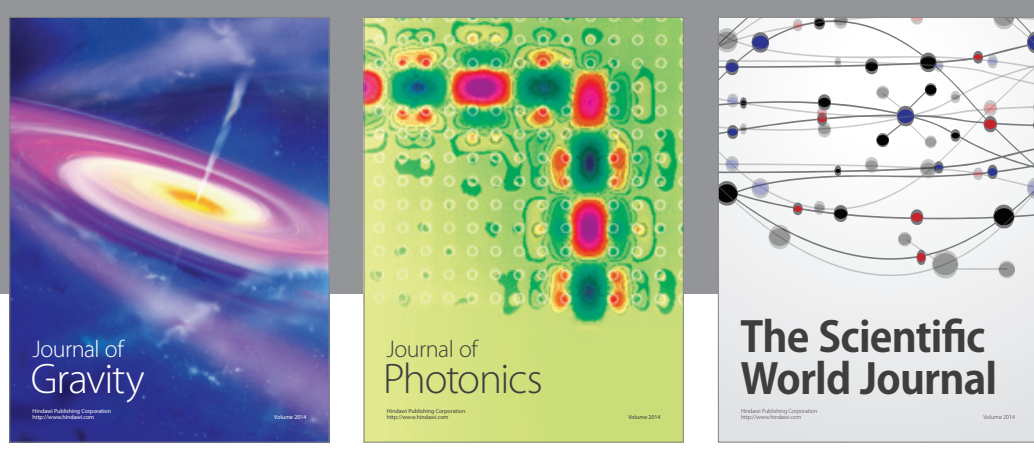

The Scientific World Journal
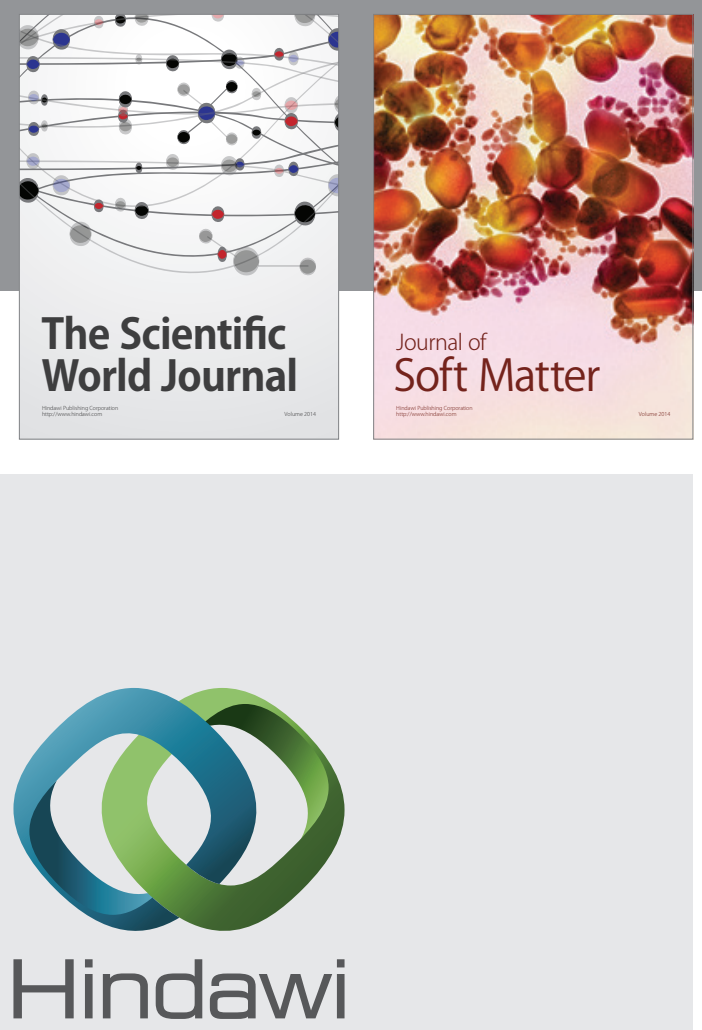

Submit your manuscripts at

http://www.hindawi.com

nternational Journal of

Statistical Mechanics
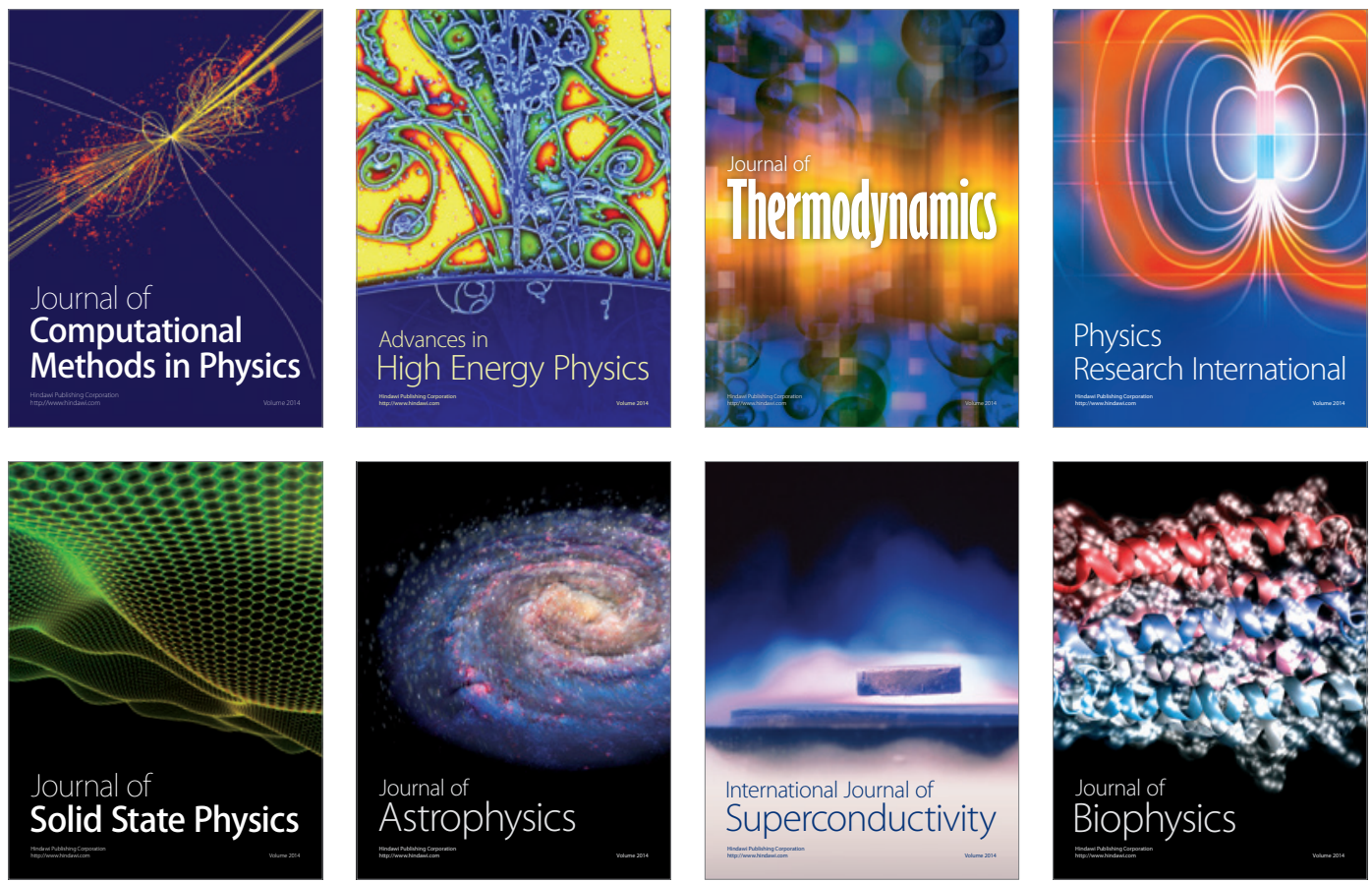
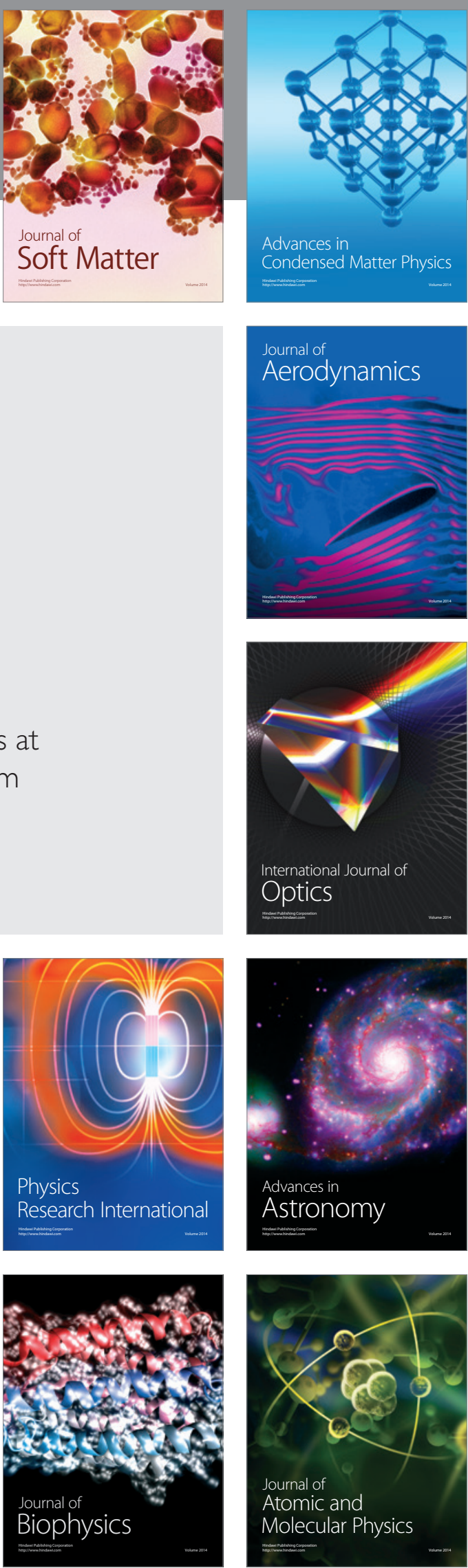